\title{
The event horizon of Sagittarius A*
}

\section{Citation}

Broderick, Avery E., Abraham Loeb, and Ramesh Narayan. 2009. The event horizon of Sagittarius $A^{*}$. The Astrophysical Journal 701, no. 2: 1357-1366. doi:10.1088/0004-637x/701/2/1357.

\section{Published Version}

10.1088/0004-637x/701/2/1357

\section{Permanent link}

http://nrs.harvard.edu/urn-3:HUL.InstRepos:27231583

\section{Terms of Use}

This article was downloaded from Harvard University's DASH repository, and is made available under the terms and conditions applicable to Other Posted Material, as set forth at http:// nrs.harvard.edu/urn-3:HUL.InstRepos:dash.current.terms-of-use\#LAA

\section{Share Your Story}

The Harvard community has made this article openly available.

Please share how this access benefits you. Submit a story.

Accessibility 


\title{
THE EVENT HORIZON OF SAGITTARIUS A*
}

\author{
Avery E. Broderick ${ }^{1}$, Abraham Loeb ${ }^{2}$, And Ramesh Narayan ${ }^{2}$ \\ ${ }^{1}$ Canadian Institute for Theoretical Astrophysics, 60 St. George St., Toronto, ON M5S 3H8, Canada; aeb@ cita.utoronto.ca \\ ${ }^{2}$ Institute for Theory and Computation, Harvard University, Center for Astrophysics, 60 Garden St., Cambridge, MA 02138, USA \\ Received 2009 March 3; accepted 2009 June 22; published 2009 July 31
}

\begin{abstract}
Black hole event horizons, causally separating the external universe from compact regions of spacetime, are one of the most exotic predictions of general relativity. Until recently, their compact size has prevented efforts to study them directly. Here we show that recent millimeter and infrared observations of Sagittarius $\mathrm{A}^{*}\left(\mathrm{Sgr} \mathrm{A}^{*}\right)$, the supermassive black hole at the center of the Milky Way, all but require the existence of a horizon. Specifically, we show that these observations limit the luminosity of any putative visible compact emitting region to below $0.4 \%$ of Sgr A*'s accretion luminosity. Equivalently, this requires the efficiency of converting the gravitational binding energy liberated during accretion into radiation and kinetic outflows to be greater than $99.6 \%$, considerably larger than those implicated in Sgr A*, and therefore inconsistent with the existence of such a visible region. Finally, since we are able to frame this argument entirely in terms of observable quantities, our results apply to all geometric theories of gravity that admit stationary solutions, including the commonly discussed $f(R)$ class of theories.
\end{abstract}

Key words: black hole physics - Galaxy: center - infrared: general - techniques: interferometric

Online-only material: color figures

\section{INTRODUCTION}

The Schwarzschild metric presents the first example of a compact horizon: an imaginary surface delineating a compact region from which the rest of the universe is causally disconnected. Subsequently, a substantial theoretical effort was made to determine if such "black hole" solutions could practically come into existence (Oppenheimer \& Snyder 1939; Penrose 1965; Wheeler 1966; Heger et al. 2003). Despite Einstein's misgivings, black holes are now believed to be the inevitable consequence of the demise of massive stars. In recent years, the existence of compact horizons has taken on a renewed significance due to efforts to construct a grand unified theory. Specifically, horizons play prominently in the well known "information paradox" (Hawking 2005; Mathur 2009). However, now we are in a position to constrain the existence of horizons observationally.

For astrophysical purposes, the critical features of horizons are (1) their compactness, allowing substantial amounts of gravitational binding energy to be liberated during accretion, and (2) their ability to hide the ultimate fate of accreting matter. Unlike a black hole, into which the kinetic and thermal energy gained during infall can disappear (adding only to the mass), objects with surfaces (e.g., stars) radiate the residual energy not emitted during the accretion process. This fact has been used in a number of efforts to test for the presence of horizons in a variety of black hole systems (Narayan et al. 1997; Narayan \& Heyl 2002; McClintock et al. 2004; Broderick \& Narayan 2006, 2007; Narayan \& McClintock 2008). For many black hole candidates the tell-tale sign of surface emission, seen in accreting neutron stars, is absent, implying the lack of an analogous surface in these objects.

Sagittarius $A^{*}\left(\operatorname{Sgr} A^{*}\right)$, the radio point source associated with the dark mass located at the center of the Milky Way, is the best studied black hole candidate to date. Near-infrared (NIR) observations of massive stars in its vicinity have provided direct mass and distance measurements, $M=(4.5 \pm 0.4) \times 10^{6} M_{\odot}$ and $D=8.4 \pm 0.4 \mathrm{kpc}$, respectively, and confined it to within $40 \mathrm{AU}$ (Ghez et al. 2008; Gillessen et al. 2009). With a luminosity of $10^{36} \mathrm{erg} \mathrm{s}^{-1}$, it is substantially underluminous relative to its limiting Eddington luminosity, $6 \times 10^{44} \mathrm{erg} \mathrm{s}^{-1}$. The emission is strongly nonthermal, distributed from the radio to $\gamma$-rays, and believed to be powered by the release of gravitational binding energy by accreting gas.

Due to its mass and proximity, Sgr A* has the largest angular size of any known black hole and offers the best prospects for direct imaging of its silhouette (Falcke et al. 2000; Broderick \& Loeb 2005, 2006a, 2006b). Unfortunately, millimeter imaging alone will be unable to verify or exclude the presence of a horizon (Broderick \& Narayan 2006). This is because even a relatively bright central object (e.g., one radiating thermally with a luminosity comparable to $\dot{M} c^{2}$ ) will appear in silhouette against the much hotter surrounding accretion flow. However, here we show that recent millimeter-Very Long Baseline Interferometric (VLBI) observations, which have resolved sub-horizon scale structure for the first time (Doeleman et al. 2008), provide conclusive evidence for the presence of a horizon when coupled with the existing NIR and mid-infrared (MIR) flux limits (Ghez et al. 2005; Hornstein et al. 2007; Schödel et al. 2007). We do this by explicitly excluding emission from a putative compact infrared photosphere, lying inside of the millimeter-emitting region. Specifically, we show that for such surface emission to remain undetected would require unphysically large radiative efficiencies, greater than $99.6 \%$, as compared to the typical efficiencies in active galactic nuclei (AGNs) of $10 \%$ (Kato et al. 2008) and the meager efficiency implicated in Sgr A*, $0.01 \%-1 \%$ (Narayan \& McClintock 2008). Most importantly, we do this using only observable quantities, ensuring that these limits are largely independent of the gravitational theory employed. ${ }^{3}$

Section 2 describes the underlying physical assumptions and Section 3 presents the recent observational constraints, within

\footnotetext{
3 Our results hold for any geometric gravitational theory admitting stationary solutions. This includes all of the $f(R)$ theories as well as black hole

alternatives within the context of general relativity, such as clusters of compact objects, boson stars, and gravastars.
} 
the context of Sgr A*. Conclusions are collected in Section 4. Appendices A and B discuss photon propagation times, the notion of gravitational binding energy, and the apparent size of objects within the context of general geometric theories of gravity.

\section{PHYSICAL ASSUMPTIONS}

Our argument depends critically upon three underlying, physically well motivated assumptions: (1) Sgr A* is accretion powered; (2) has reached steady state; and (3) compact surfaces are approximate blackbodies. We wish to emphasize that relaxing any of these would require fundamental alterations to presently well understood physics (such as microscopic physics at pedestrian, and laboratory accessible, densities, temperatures, and magnetic field strengths) or the invention of exotic new states of matter. Nevertheless, we discuss each in detail, describing how these are justified in the context of Sgr A*.

\subsection{Accretion Power and the Luminosity of Sgr A*}

It has been widely accepted that the emission from Sgr A* is powered by accretion. This is inferred from a variety of sources, including the spectrum, variability, VLBI observations, environment, and polarization of Sgr A*. Here we review these, if only to list the numerous observational hurdles facing any alternate interpretation.

The spectrum of Sgr A* extends from the radio to the gammarays, peaking in the submillimeter, where it transitions from a featureless, inverted power-law to an optically thin spectrum. Sgr A*'s emission is strongly nonthermal and exhibits no absorption lines, which despite its low bolometric luminosity (comparable to a B-star) rules out a hydrostatic, optically thick gas cloud, similar to a large star. Furthermore, this spectrum is very similar to that from other AGNs, for which there is no known alternative power source with the necessary efficiency.

At centimeter wavelengths and below, Sgr A* exhibits considerable short timescale variability. Flares have been observed in the millimeter, submillimeter, infrared, and X-ray bands, with rise times and variability timescales comparable to the periods of innermost stable orbits around general relativistic (GR) black holes. At considerably longer wavelengths, where $\mathrm{Sgr} \mathrm{A}^{*}$ is optically thick, the degree of variability decreases and the characteristic timescales increase. This is consistent with a stratified emitting region, with short-wavelength emission arising close to the central mass.

This interpretation is confirmed by VLBI observations. Observations with the Very Long Baseline Array at wavelengths from $3 \mathrm{~mm}$ to $6 \mathrm{~cm}$ have resolved the intrinsic size of the radio emitting region about Sgr A* (Bower et al. 2006; Shen et al. 2005; Krichbaum et al. 2006). This is somewhat complicated by interstellar scattering, requiring a careful subtraction of the scattering law determined by fitting the observed size of Sgr A* from $6 \mathrm{~cm}$ to $24 \mathrm{~cm}$. While there is some debate over the precise form of the scattering law, there is no question that Sgr A* is indeed radially stratified, with the radius of the photosphere decreasing with decreasing wavelength.

Turning to its environment, Sgr A* suffers from an embarrassment of riches. The stellar winds from nearby massive stars $\left(\simeq 0.1 \mathrm{pc}\right.$ from $\left.\mathrm{Sgr} \mathrm{A}^{*}\right)$ provide sufficient material to support luminosities more than 11 orders of magnitude greater than that observed. Indeed, the difficulty in modeling Sgr A* has been to explain its meager luminosity instead of the prodigious output predicted. This has typically been done by postulating an extraordinarily low radiative efficiency and/or the existence of accretion-powered outflows (Narayan et al. 1995; Blandford \& Begelman 1999).

Finally, below roughly $3 \mathrm{~mm}, \mathrm{Sgr} \mathrm{A}^{*}$ is linearly polarized (Aitken et al. 2000; Bower et al. 2003; Macquart et al. 2006; Marrone et al. 2006, 2007). At longer wavelengths it is Faraday depolarized, providing some measurement of the density and magnetic field strength near the central mass. Assuming near equipartition magnetic fields, this implies a density of cold electrons of roughly $10^{6} \mathrm{~cm}^{-3}$ at radii of $10^{13}-10^{14} \mathrm{~cm}$ (Agol 2000; Quataert \& Gruzinov 2000; Marrone et al. 2006). Assuming stronger, large-scale magnetic fields results in lower density estimates, and conversely, large amounts of magnetic turbulence results in higher estimates. While this does not provide a direct measurement of the accretion rate, it is difficult to imagine that the magnetorotational instability is incapable of driving accretion in this environment at rates up to $10^{-8} \dot{M}_{\odot} \mathrm{yr}^{-1}$.

\subsection{Steady State}

Assuming steady state will allow us to relate the surface emission from black hole alternatives to that from the accretion flow. Within the context of Sgr A* there are a variety of reasons to expect that any black hole alternative will have reached some sort of steady state (see Broderick \& Narayan 2007). However, we point out that black holes explicitly violate this condition: the unradiated kinetic energy is advected across the horizon and then added to the mass of the black hole.

The dynamical timescale of Sgr A* depends upon the nature of the object. Nevertheless, we may expect that it is comparable to the dynamical timescale of the corresponding black hole, $G M / c^{3} \simeq 20$ s. This is supported by the $\sim 10$ min variability observed in the NIR and X-rays, presumed to be associated with material orbiting nearby. Both of these are much, much shorter than the estimated age of Sgr A* $10 \mathrm{Gyr}$, making it natural to assume that it has sufficient time to reach steady state. Note that if a substantial portion of Sgr A*'s mass was accrued via accretion, its accretion rate must have been much larger at times in the past. Thus if the timescale for reaching steady state exceeds the period since the previous active phase we will be underestimating Sgr A*'s luminosity.

While the extreme difference in timescales naturally implies that even surfaces with extraordinary redshifts $\left(z \lesssim 10^{15}\right)$ will have reached steady state, within the context of black hole alternatives exhibiting GR exterior spacetimes a much stronger statement can be made. This is because photons that are emitted initially outward and subsequently lensed back onto Sgr $\mathrm{A}^{*}$ necessarily provide a mechanism to couple otherwise disparate regions of the photosphere. The travel times of these photons diverge only logarithmically with photosphere redshift, and therefore place an upper bound upon the equilibration timescale.

Inherent in these arguments is the assumption that locally the surface properties of any black hole alternative are not illbehaved. That is, the self-coupling is what determines the equilibration timescale. This may not be the case if, e.g., the heat capacity of the surface is effectively nearly infinite (or as in the case of a black hole, negative). However, if the rate at which accreted baryonic material is incorporated into the surface is sufficiently slow, a baryonic atmosphere will develop. In this case, even for objects with near-vanishing temperature a hot atmosphere can be produced which satisfies the steady-state condition.

\subsection{Compact Objects and Blackbodies}

Our argument will hinge upon our ability to specify the spectral signatures of any surface emission from black hole 


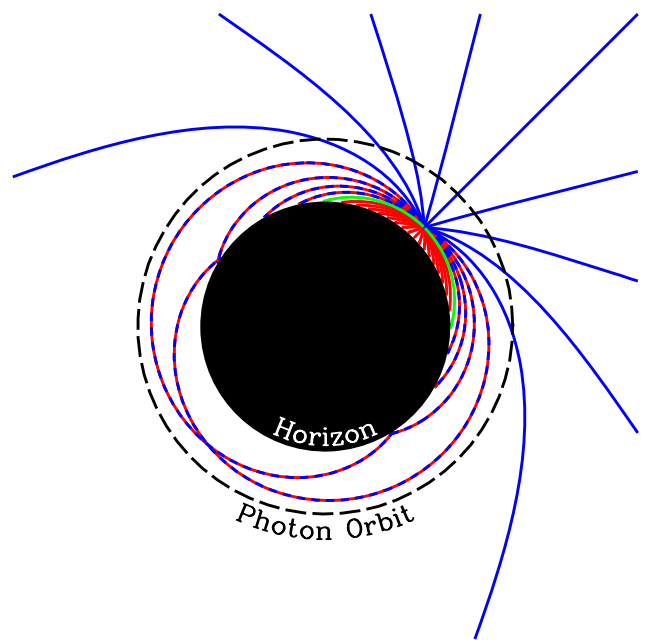

Figure 1. Rays launched isotropically (every $10^{\circ}$ ) in the locally flat, stationary frame are lensed in a Schwarzschild spacetime. Those rays that are initially moving inward, tangentially and outward are shown in red, green, and blue, respectively. Additionally, those that are launched initially moving outward and are subsequently captured are red-blue dashed. For reference the horizon and photon orbit are shown. Generically, the fraction of rays that escape to infinity decreases as the emission point is moved toward the black hole, dropping below $50 \%$ at the photon orbit and dropping all the way to $0 \%$ at the horizon. As a consequence of this strong lensing, emitting objects that are contained within the photon orbit approximate the canonical pin-hole cavity example of a blackbody, becoming a perfect blackbody in the limit that the surface redshift goes to $\infty$. (A color version of this figure is available in the online journal.)

alternatives. Subject to the condition outlined in Section 2.2, it is natural to expect any such emission to be thermal. This is obvious if the object accrues an optically thick atmosphere of baryonic material. However, for any compact surface which lies within the photon orbit this is generally an excellent assumption.

By definition, at the photon orbit whether or not a photon impacts the surface is determined by the radial component of the momentum. Thus, if photons are emitted isotropically by a surface located at the photon orbit, half will impact the surface and half will escape to infinity. As the surface shrinks inside the photon orbit the fraction of escaping photons also decreases, falling to zero when the surface coincides with the horizon. ${ }^{4}$ Thus, the higher the redshift of the surface, the more effectively different regions on the surface are coupled to one another via radiation and the closer the surface approaches thermodynamic equilibrium. Consequently, highredshift surfaces present a perverse realization of the canonical pin-hole cavity, becoming ideal blackbodies as $z$ goes to $\infty$ (Broderick \& Narayan 2006). For a Schwarzschild spacetime this is shown in Figure 1; however, this behavior is generic to spherically symmetric spacetimes. Thus if the system has sufficient time to have reached steady state, it must be a blackbody.

\footnotetext{
4 Note that this implies that photon orbits exist for all spherically symmetric spacetimes that admit horizons. Consider a population of photons emitted such that they are initially locally isotropic. At infinity the fraction of escaping photons is unity, while at the horizon it vanishes. Thus, we are guaranteed that at some location between the two there exists a point where only half escape. Due to the symmetry of the spacetime, it can be shown that this necessarily coincides with a closed photon orbit. For our purpose this simply defines a convenient scale for the definition of a "compact" surface.
}

\section{OBSERVATIONAL LIMITS UPON THE EXISTENCE OF HORIZONS}

The primary astrophysical importance of a horizon is that the gravitational binding energy liberated by material as it accretes can be advected into the black hole without any further observational consequence. This is very different from accretion onto other compact objects, e.g., neutron stars, in which this liberated energy ultimately must be emitted by the stellar surface. Importantly, this argument is not dependent upon the particulars of the compact object. Any object powered by accretion, whose surface is visible from the external universe, should show evidence of surface radiation. We will use this fact to rule out the possibility that accreted material in Sgr A* settles in a region visible to outside observers, and in doing so make the argument that a horizon must exist. That is, we imagine that material comes to rest at some surface where it radiates its remaining kinetic energy and observationally constrain the associated surface luminosity.

The gravitational binding energy released by a particle falling onto the putative surface depends upon the details of the gravitational theory. For those theories which admit notions of energy conservation (in the test particle limit), including all stationary spacetimes, we may write this in terms of the specific binding energy at the putative surface, $\Delta \epsilon_{g}$. That is, the liberated energy due to a particle of mass $m$ as measured at infinity is $E_{\infty}=\Delta \epsilon_{g} m c^{2}$. For a continuous accretion flow, this provides a total power, as measured at infinity, of

$$
L_{\infty}=\Delta \epsilon_{g} \dot{M} c^{2},
$$

where $\dot{M}$ is the mass accretion rate at the surface.

The particular form of $\Delta \epsilon_{g}$ also depends upon the nature of the accretion flow. For arbitrary stationary, spherically symmetric spacetimes this is computed in Appendix A for two typical cases: zero-angular momentum accretion flows in which matter does not orbit the black hole (e.g., Bondi accretion) and Keplerian accretion disk (e.g., appropriate for thin disks). In the latter case $\Delta \epsilon_{g}$ is generally smaller since a fraction of the liberated binding energy is necessarily converted into the kinetic energy associated with the orbital motion. Within the context of GR black holes ${ }^{5} \Delta \epsilon_{g}$ is straightforward to compute explicitly for these cases, and is shown in Figure 2 for both nonrotating and rapidly rotating black holes. However, we shall see that our ultimate constraints are independent of the form of $\Delta \epsilon_{g}$.

Only a fraction of $L_{\infty}$ is converted into radiation. The observed electromagnetic luminosity at infinity may be parameterized in terms of a radiative efficiency, $\eta_{r}$ :

$$
L_{\mathrm{obs}}=\eta_{r} L_{\infty}
$$

Gravitational binding energy may also be converted into the kinetic energy of relativistic outflows, which we similarly parameterize in terms of outflow efficiency, $\eta_{k}$ :

$$
L_{\text {out }}=\eta_{k} L_{\infty} .
$$

It is important to note that $\eta_{r}$ and $\eta_{k}$ are primarily a function of the accretion flow, dependent upon the microphysics, and thus relatively insensitive to the character of strong gravity.

\footnotetext{
5 Throughout this section we will punctuate the analysis with examples from GR black holes. However, it should be understood that our analysis is independent of these specific examples, and in fact considerably more general.
} 


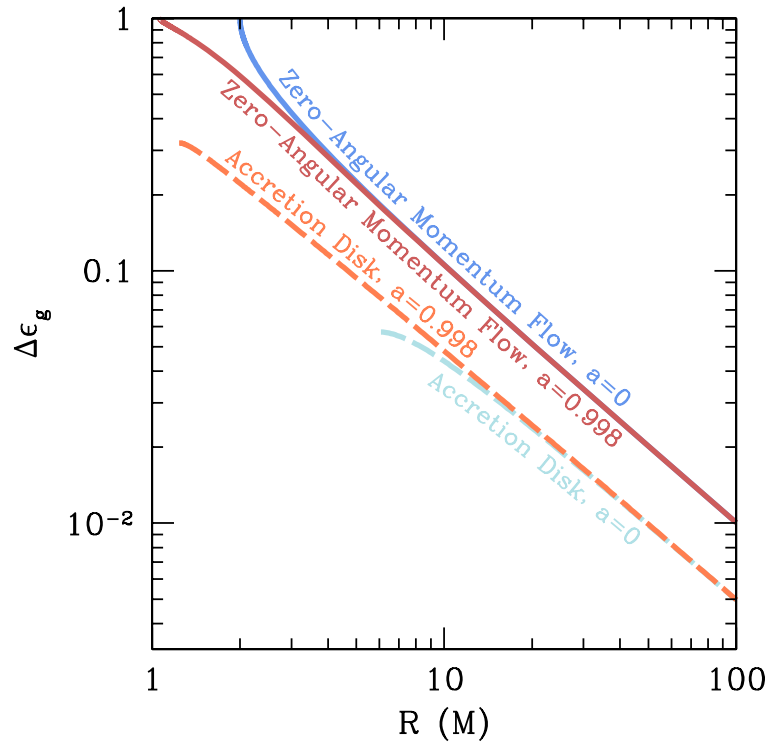

Figure 2. Left: binding energy released per unit rest mass as a function of radius for material that has come to rest in the zero-angular momentum frame (solid lines) and the Keplerian frame (dashed lines) around a GR black hole. This is shown for a nonrotating GR black hole (Schwarzschild) by the blue lines and for a rapidly rotating ( $a=0.998$ ) GR black hole (Kerr) by the orange lines. Note that in both cases, for the zero-angular momentum flow the entire rest mass is released at the horizon, while the energy released by an accretion disk peaks at the ISCO (inside of which no further stable orbits exist). Finally, beyond approximately $10 \mathrm{M}$, both follow their Newtonian expressions.

(A color version of this figure is available in the online journal.)

If a horizon is present, the remainder of $L_{\infty}$ may be advected across it without further observational consequence. However, in the presence of a surface, if $\mathrm{Sgr} \mathrm{A}^{*}$ has reached steady state, this remainder must ultimately be radiated. Thus the surface luminosity, as measured at infinity, is

$$
L_{\text {surf }}=L_{\infty}-L_{\mathrm{obs}}-L_{\mathrm{out}}=\frac{1-\eta_{r}-\eta_{k}}{\eta_{r}} L_{\mathrm{obs}},
$$

where we have written this entirely in terms of the unknown efficiencies and the observed luminosity. (Specifically, note that neither $\Delta \epsilon_{g}$ nor $\dot{M}$ appears in this expression.)

As we have argued in Section 2.3, for compact surfaces this radiation will be in the form of a blackbody spectrum. That is, in terms of the apparent radius $\left(R_{a}\right)$ and temperature $\left(T_{\infty}\right)$ of the putative surface, as measured at infinity,

$$
L_{\text {surf }}=4 \pi \sigma R_{a}^{2} T_{\infty}^{4} .
$$

Alternatively, this provides a means to estimate the surface temperature (and thus spectrum) given $L_{\text {surf }}$ and $R_{a}$. Combined with Equation (4), we may write $T_{\infty}$ in terms of $L_{\mathrm{obs}}, R_{a}, \eta_{r}$, and $\eta_{k}$ :

$$
T_{\infty}=\left(\frac{1-\eta_{r}-\eta_{k}}{\eta_{r}} \frac{L_{\mathrm{obs}}}{4 \pi \sigma R_{a}^{2}}\right)^{1 / 4} .
$$

In terms of this surface temperature, the expected flux seen by distant observers is then

$$
F_{v}=\pi\left(\frac{R_{a}}{D}\right)^{2} B_{v}\left(T_{\infty}\right),
$$

where $B_{v}$ is the blackbody spectrum.

However, no thermal surface component is observed in Sgr A*'s spectrum. Thus, if such surface emission is present, it must be hidden under the emission from the accretion flow. As a consequence, each flux measurement constitutes an independent upper limit upon the surface flux, and thus surface temperature. Explicitly, given an observed flux $F_{v}^{\text {obs }}$, $T_{\infty} \leqslant T_{\max }\left(v, F_{v}^{\text {obs }}, R_{a} / D\right)$ where

$$
T_{\max }\left(v, F_{v}^{\mathrm{obs}} ; \frac{R_{a}}{D}\right)=h v / k \ln \left(1+\frac{2 \pi h v^{3} R_{a}^{2}}{c^{2} F_{v}^{\mathrm{obs}} D^{2}}\right) .
$$

Note that this is generally a function of $R_{a}$, a consequence of the fact that larger surfaces are correspondingly cooler, and therefore easier to hide under the observed emission.

This maximum temperature then implies a limit upon $L_{\text {surf }}$ directly via the blackbody condition:

$$
\frac{L_{\text {surf }}}{L_{\text {obs }}} \leqslant \frac{L_{\text {surf, max }}}{L_{\mathrm{obs}}} \equiv \frac{\sigma R_{a}^{2}}{D^{2} F_{\mathrm{obs}}} T_{\max }^{4}\left(v, F_{v}^{\mathrm{obs}} ; \frac{R_{a}}{D}\right),
$$

where $F_{\text {obs }}=L_{\text {obs }} / 4 \pi D^{2}$ is the integrated observed flux from Sgr A*. Alternatively, we may rewrite this in terms of a lower limit upon the efficiencies:

$$
\eta_{r}+\eta_{k} \geqslant \frac{1}{1+L_{\text {surf,max }} / L_{\text {acc }}} .
$$

That is, if the efficiencies are sufficiently high the unradiated liberated energy in the accretion flow is sufficiently small that the surface could escape detection. Unlike $L_{\text {surf }} / L_{\text {acc }}$, we have natural scales against which to compare $\eta_{r}+\eta_{k}$. In prodigiously accreting systems, such as AGNs, X-ray binaries, and gammaray bursts, which are believed to be radiatively efficient, the radiative efficiency $\eta_{r}+\eta_{k}$ is estimated to be $\sim 10 \%$ (Kato et al. 2008). In Sgr $\mathrm{A}^{*}$, on the other hand, $\eta_{r}$ is thought to be quite small $(0.01 \%-1 \%$ for typical accretion models) as a consequence of the weak coupling between accreting ions and electrons within the gas (Narayan \& McClintock 2008), and there is presently no direct evidence for energetic outflows.

Within the context of GR black holes and standard accretion theory, there are fundamental limits upon how large $\eta_{r}+\eta_{k}$ may be for compact surfaces. This arises from the fact that matter inside of the innermost stable circular orbit (ISCO) plunges rapidly onto the surface, and thus does not have sufficient time to radiate. In contrast, outside of the ISCO material may linger on stable orbits for long periods of time, and at least in principle can radiate efficiently. Thus, even if the "intrinsic" radiative efficiency outside of the ISCO reached unity, the accreting material would still accrue additional kinetic energy during the plunge from the ISCO to the putative surface. If this surface is located inside of the ISCO, we may therefore place a lower bound upon the additionally liberated energy, and thus an upper bound upon $\eta_{r}+\eta_{k}$. These are shown in Figure 3 for zeroangular momentum accretion flows ${ }^{6}$ and orbiting accretion disks surrounding both nonrotating and rapidly rotating black holes (see Appendix A for details). For orbiting disks the maximum value of $\eta_{r}+\eta_{k}$ is generally less than $33 \%$.

Therefore, each combined size and flux measurement of Sgr $\mathrm{A}^{*}$ places a direct constraint upon the luminosity of a putative surface, or, equivalently, upon the radiative efficiency of the accretion flow. Thus, we now turn our attention to describing the current best constraints upon $R_{a} / D$ and $F_{v}^{\text {obs }}$.

\footnotetext{
6 Note that we are being maximally conservative by assuming that a zero-angular momentum flow can radiate all its binding energy down to the ISCO. Models of nonrotating accretion flows are usually very radiatively inefficient. Allowing for this would strengthen our argument substantially.
} 


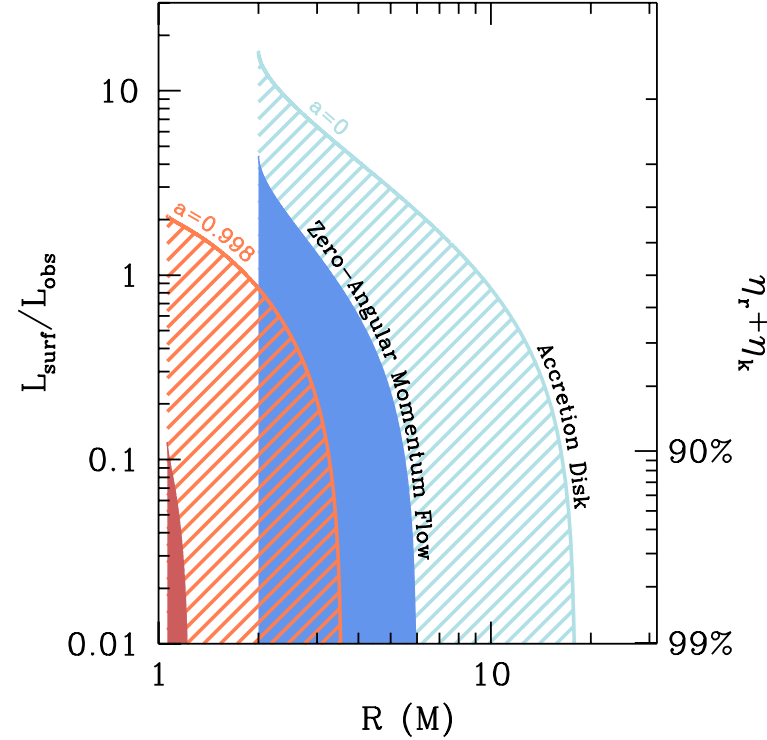

Figure 3. Constraints upon $L_{\text {surf }} / L_{\text {obs }}$ (or $\eta_{r}+\eta_{k}$ ) in GR black hole spacetimes as a function of the coordinate radius of the stopping region. In the shaded/hatched regions $\eta_{r}+\eta_{k}>\Delta \epsilon_{g}(R) / \Delta \epsilon_{g}\left(r_{\text {ISCO }}\right)$ and are thus not possible in the context of GR accretion theory. This is shown for when the accreted material comes to rest in the zero-angular momentum frame and the Keplerian frame around nonrotating and rapidly rotating $(a=0.998)$ black holes. In all cases, the shaded regions are truncated at the coordinate position of the relevant horizons and the excluded $L_{\text {surf }} / L_{\text {obs }}$ vanishes at some radii, which is a natural result of fixing $r_{\text {ISCO }}$ (for the zero-angular momentum flow, this happens at $r_{\text {ISCO }}$ itself). (A color version of this figure is available in the online journal.)

Table 1

VLBI Size Constraints

\begin{tabular}{lccc}
\hline \hline$\lambda(\mathrm{mm})$ & $R_{a}(\mu \mathrm{as})^{\mathrm{a}}$ & $3 \sigma R_{a}(\mu \mathrm{as})^{\mathrm{b}}$ & Reference \\
\hline 1.3 & 19 & 27 & Doeleman et al. (2008) \\
3 & 63 & 84 & Shen et al. (2005) \\
7 & 120 & 150 & Bower et al. (2004) \\
\hline
\end{tabular}

Notes.

${ }^{a}$ The measured half-width, half-max.

$\mathrm{b}$ The $3 \sigma$ upper limit upon the half-width, half-max.

\subsection{Millimeter Size Constraints}

Measuring the intrinsic size of $\mathrm{Sgr} \mathrm{A}^{*}$ is complicated by its low luminosity, exceedingly small size, interstellar scattering and the opacity of the surrounding accretion flow. As a result, high-frequency VLBI has produced the only meaningful limits upon the size of a putative surface. Recent measurements of the photosphere radius (half-width, half-max) are listed in Table 1. As we shall see in the following section, the emission from a putative surface will typically peak in the NIR, where Sgr A* is completely optically thin. As a consequence, the size limits estimated via VLBI are likely to be overestimates of a putative surfaces extent.

At wavelengths longer than $1.3 \mathrm{~mm} \mathrm{Sgr} \mathrm{A*} \mathrm{is} \mathrm{optically} \mathrm{thick}$ and the observed size is dominated by interstellar scattering, requiring a careful subtraction of the empirically determined scattering law. However, at $1.3 \mathrm{~mm}$ interstellar scatting is subdominant and the plasma surrounding Sgr $\mathrm{A}^{*}$ has become optically thin. Perhaps surprisingly, at this wavelength the inferred angular size, $37 \pm 11 \mu$ as, is smaller than the apparent diameter of the horizon (48-55 $\mu$ as depending upon spin), independent of black hole spin (Doeleman et al. 2008). However, this is natural in the context of an orbiting accretion flow
Table 2

Dereddened Infrared Flux Limits

\begin{tabular}{lccc}
\hline \hline Band & $\lambda(\mu \mathrm{m})$ & $F_{\nu}(\mathrm{mJy})^{\mathrm{a}}$ & Reference \\
\hline$H$ & 1.6 & $2.60 \pm 0.28$ & Hornstein et al. (2007) \\
$K^{\prime}$ & 2.1 & $1.66 \pm 0.18$ & Hornstein et al. (2007) \\
$L^{\prime}$ & 3.8 & $1.28 \pm 0.30$ & Ghez et al. (2005) \\
$M_{S}$ & 4.7 & $2.2 \pm 1.6$ & Hornstein et al. (2007) \\
$N$ & 8.7 & $22 \pm 14$ & Schödel et al. (2007) \\
\hline
\end{tabular}

Note. ${ }^{\text {a }}$ The uncertainty in $F_{v}$ is taken to be the $1 \sigma$ detection uncertainty.

or an outflow; in both cases the image is dominated by the approaching plasma (Broderick et al. 2009; Broderick \& Loeb 2009). Therefore, the measured $37 \pm 11 \mu$ as may not represent $R_{a} / D$ of the surface itself, but instead result from the velocity structure of the accreting material. Nevertheless, efforts to fit this result using existing accretion models for Sgr A* imply that the surface lies within the photon orbit.

\subsection{Infrared Flux Limits}

Given the size limits, typical values of $T_{\infty}$ range from $10^{2}$ $10^{4} \mathrm{~K}$, and thus the emission from a putative surface peaks in the near and mid-infrared. Fortunately, despite the large infrared extinction in the direction of the Galactic center, there are a number of observations of Sgr A* at these wavelengths. Furthermore, there exists an accurate empirically determined extinction law for this region (Moneti et al. 2001), making it possible to deredden the observed fluxes, producing intrinsic infrared flux measurements for Sgr A*.

However, in these bands the emission from Sgr $\mathrm{A}^{*}$ is dominated by variability (see, e.g., Hornstein et al. 2007), which must clearly be associated with the accretion flow and not the surface. This prevents an unambiguous detection of quiescent emission. Therefore, the flux limits we collect in Table 2 are determined by inspecting variable infrared light curves and extracting the lowest flux detection. The $L^{\prime}(3.8 \mu \mathrm{m})$ and $N$ $(8.7 \mu \mathrm{m})$ band limits are taken directly from Ghez et al. (2005) and Schödel et al. (2007), respectively. The $H(1.6 \mu \mathrm{m}), K^{\prime}$ $(2.1 \mu \mathrm{m})$, and $M_{S}(4.8 \mu \mathrm{m})$ band limits were determined by examining the panels of Figure 2 of Hornstein et al. (2007), which show dereddened flux light curves for a number of infrared flares in Sgr A* and a variety of near-infrared bands.

\subsection{Constraints Upon Surface Existence}

Each of the infrared flux limits listed in Table 2 places an upper limit upon $L_{\text {surf }} / L_{\mathrm{obs}}$ via Equation (9), given a value of $R_{a}$. Figure 4 shows these limits as a function of surface size, together with their $3 \sigma$ upper bounds (denoted by the hatched regions). Generally, larger surfaces are cooler. This has two consequences. First, since the strongest constraints are placed by flux measurements near the peak in the thermal spectrum, the infrared band providing the most stringent limit is a function of $R_{a}$ as well; near $R_{a} / D \simeq 100 \mu$ as the infrared band dominating the constraint changes from $M_{S}(4.7 \mu \mathrm{m})^{7}$ to $N(3.8 \mu \mathrm{m})$. The constraint due to the combination of all of the infrared bands is the lower envelope of all the bands, and defines the excluded region, corresponding to small, luminous surfaces. Second, since cooler surfaces are more easily masked by the emission from the accretion flow, the limit becomes less stringent as $R_{a}$

\footnotetext{
7 The small uncertainties associated with the $L^{\prime}(3.8 \mu \mathrm{m})$ measurement dominate the uncertainties at low $R_{a}$ despite the fact that the limit derived from the best estimate of the $M_{S}$-band flux is always stronger.
} 


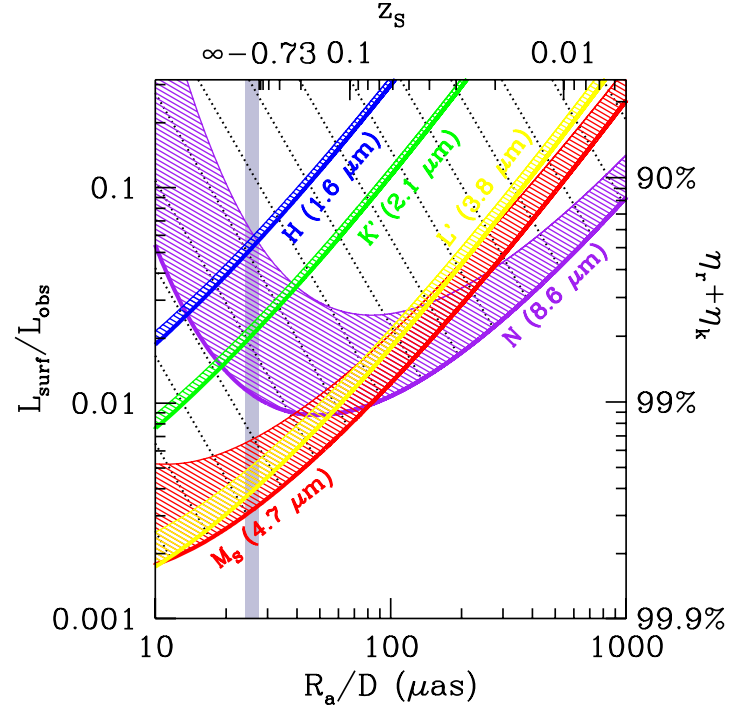

Figure 4. Limits upon $L_{\text {surf }} / L_{\text {obs }}$ implied by Equation (9) as a function of the photosphere size as seen at infinity for the infrared measurements listed in Table 2. The hatched bands denote the $3 \sigma$ upper bounds. The peculiar behavior of the $\mathrm{N}$-band constraint is a result of the transition from the Rayleigh-Jeans limit to the Wien limit around $R_{a} / D \simeq 50 \mu$ as as the surface becomes cooler. The region above any of the limits is necessarily excluded. The right-hand vertical axis shows the corresponding limits upon the accretion flow's radiative efficiencies. The top axis gives the redshift associated with a Schwarzschild spacetime given the apparent source radius and the thick gray line shows the apparent radii associated with the photon orbit for Kerr spacetimes.

(A color version of this figure is available in the online journal.)

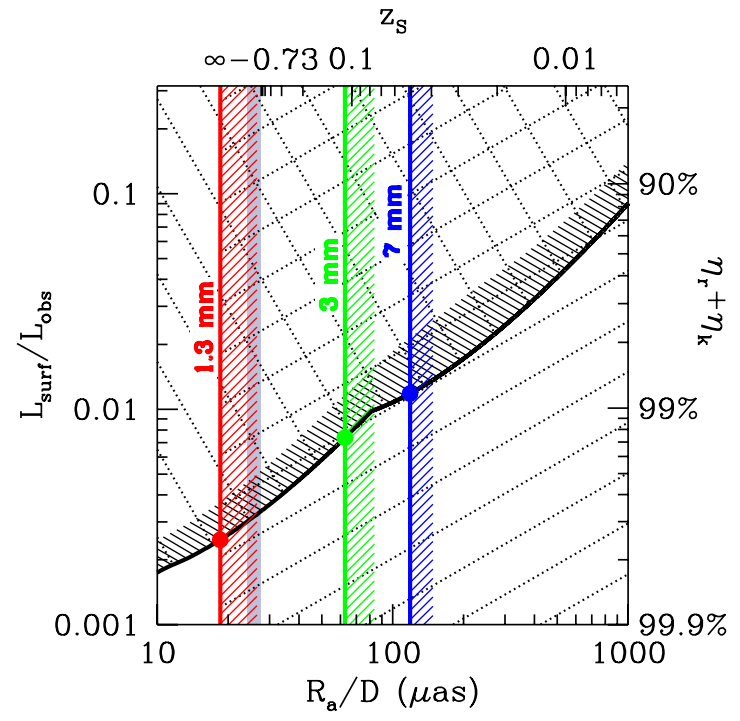

Figure 5. Limits upon $R_{a} / D$ implied by recent VLBI observations listed in Table 1, overlaid upon the combined constraint implied by IR flux measurements. Regions to the right of the left-most (smallest) size constraint are excluded. When combined with the limits from the IR flux measurements, the permissible parameter space is reduced to a small corner in the $R_{a}-L_{\mathrm{surf}} / L_{\mathrm{obs}}$ plane. The axes are identical to those in Figure 4.

(A color version of this figure is available in the online journal.)

increases. Thus, the infrared constraint upon $L_{\text {surf }} / L_{\mathrm{obs}}$ must be supplemented with an independent limit upon the size of the putative surface.

Upper limits upon $R_{a} / D$ are found directly via the radio VLBI observations collected in Table 1. These are shown in Figure 5, with their $3 \sigma$ upper bounds (again denoted by the hatched regions) together with the combined infrared limit. The recent

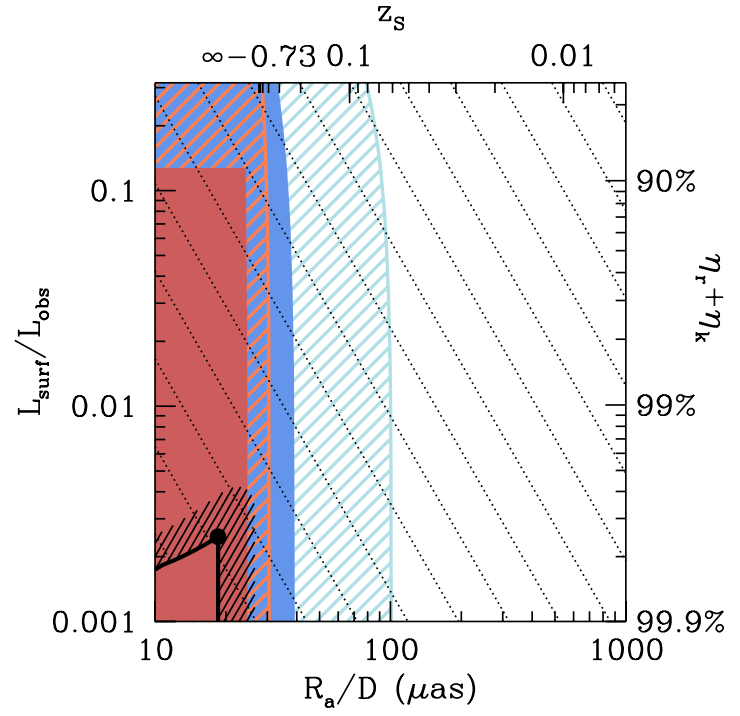

Figure 6. Combined limits upon $L_{\text {surf }} / L_{\text {obs }}$ and $R_{a} / D$ compared with the regions generally excluded in GR black hole spacetimes (shown as a function of coordinate radius in Figure 3). Extending these to angular scales smaller than the theoretical minimum (the angular size of the horizon) results in the rectangular appearance. Blue and red excluded regions correspond to efficiencies above the maximum possible values for accretion onto Schwarzschild and maximal-Kerr black holes, respectively. Hatched and solid limits correspond to Keplerian and zero-angular momentum accretion flows, respectively. Given the most recent infrared flux limits and millimeter-VLBI size constraints, $\eta_{r}+\eta_{k}$ is inconsistent by more than an order of magnitude with estimates for Sgr A* specifically, relativistic accretion flows generally and even fundamental limits in GR spacetimes. The axes are identical to those in Figure 4.

(A color version of this figure is available in the online journal.)

$1.3 \mathrm{~mm}$ detection is the strongest, and excludes $R_{a} / D>27 \mu$ as at the $3 \sigma$ level. When combined with the infrared constraints, only a small corner of the $R_{a}-L_{\text {surf }} / L_{\text {obs }}$ is still permitted, corresponding to small, dim surfaces. Earlier measurements at $7 \mathrm{~mm}$ and $3 \mathrm{~mm}$ already required $L_{\text {surf }} / L_{\text {obs }} \lesssim 0.02$. The new $1.3 \mathrm{~mm}$ detection improves these by nearly an order of magnitude, requiring $L_{\text {surf }} / l_{\text {obs }} \lesssim 0.004$ at the $3 \sigma$ level.

A more physical interpretation is provided by the accretion efficiencies the latest observations now demand, shown on the right-hand vertical axis. In order for a surface to be present $\eta_{r}+\eta_{k} \gtrsim 99.6 \%$ (at $3 \sigma$ ). That is, as matter falls onto Sgr A*, somehow $99.6 \%$ of the liberated gravitational binding energy must be radiated, powering either the observed luminosity or kinetic outflows. Otherwise the emission of the remainder upon settling onto the surface would have been detected. This needed efficiency is considerably larger than even the $10 \%$ efficiencies implicated in rapidly accreting systems, let alone the meager efficiencies $(0.01 \%-1 \%)$ inferred in Sgr A*.

More striking is the comparison of this to the maximum efficiencies within GR spacetimes. A variety of black hole alternatives are grafted onto such spacetimes outside of some compact region, and thus in these the GR limits explicitly hold. For other black hole alternatives, including those associated with different gravity theories, these limits at least provide a scale for comparison. These fundamental constraints upon the efficiencies arise from the fact that accreting material cannot radiate efficiently inside of the ISCO. Inside of the ISCO accreting material plunges inward rapidly in comparison to the radiative timescales. Thus, even if the intrinsic radiative efficiency outside the ISCO is 100\% (which is extremely unlikely), the fraction of the total binding energy released by accreting gas in the course of its inward flow is limited to that 
fraction that is emitted outside the ISCO. Therefore, within the context of GR, even with the most conservative of assumptions (zero-angular momentum accretion flow with $100 \%$ radiative efficiency down to the ISCO) we obtain an absolute upper limit on $\eta_{r}+\eta_{k}$ for surfaces which lie within the photon orbit of $91 \%$, while for a more reasonable accretion scenario involving orbiting gas the limit is $33 \%$ (see Appendix A for more details). The combined observational limits upon $L_{\text {surf }} / L_{\text {acc }}$ and $\eta_{r}+\eta_{k}$ are compared against those inferred in GR spacetimes for zero-angular momentum and Keplerian flows in Figure 6. In particular, there is no longer any allowed region given the recent $1.3 \mathrm{~mm}$ size constraint.

\section{CONCLUSIONS}

Recent infrared and millimeter-VLBI observations imply that if the matter accreting onto Sgr $\mathrm{A}^{*}$ comes to rest in a region visible to distant observers, the luminosity associated with the surface emission from this region satisfies $L_{\text {surf }} / L_{\text {acc }} \lesssim$ 0.004. Equivalently, these observations require that $99.6 \%$ of the gravitational binding energy liberated during infall is radiated in some form prior to finally settling. These numbers are inconsistent by orders of magnitude with our present understanding of the radiative properties of Sgr A*'s accretion flow specifically and relativistic accretion flows generally. Therefore, it is all but certain that no such surface can be present, i.e., an event horizon must exist.

Our conclusions rest upon three rather conservative physical assumptions: (1) Sgr A* is gravitationally powered, (2) Sgr $\mathrm{A}^{*}$ has reached an approximate steady state and (3) a putative surface would be sufficiently compact and/or optically thick to be well modeled by a thermal spectrum. Black holes evade our argument since they violate the second of these, failing ever to reach steady state and growing in mass as a consequence. Some black hole alternatives have been proposed which would also violate the steady-state condition intrinsically, though these depend upon exotic new physical models and it is likely that the baryonic atmospheres they will inevitably develop via accretion will indeed reach steady state separately. Alternatively, there do exist astronomical objections that presumably violate the first assumption, namely rotationally powered objects such as pulsars. However, since we have fundamentally constrained the luminosity of a putative surface, independent of the mechanism powering the emission, rotation powered objects must satisfy a similar constraint, though a full discussion is beyond the scope of this paper.

Critical to our analysis is the recent submillimeter constraint upon the size of the emitting region of $\mathrm{Sgr} \mathrm{A}^{*}$ since it (1) justifies the assertion that the putative photosphere is sufficiently compact to be treated as a blackbody, (2) limits the photosphere's temperature from below, and (3) within the context of GR provides a strong constraint upon possible values of the radiative efficiency that is easily excluded. Future millimeter-VLBI observations will be critical to understanding the morphology of Sgr A*'s emitting region, and thus validating the interpretation of current observations as the approaching side of an orbiting accretion flow, with the attendant implications for Sgr A*'s size. However, unless the spacetime around Sgr A* deviates substantially from that of a GR black hole, future observations of the intrinsic size of $\mathrm{Sgr} \mathrm{A}^{*}$ will be unable to further restrict the size of a putative surface due to the influence of gravitational lensing. The reason is that all objects which lie within the photon orbit generally have the same apparent radius. Thus, obtaining more sensitive near-infrared flux measurements will remain a crucial avenue for strengthening this kind of argument.

We were able to place these constraints completely in terms of observable quantities: fluxes and the apparent size of Sgr A*. Beyond using GR spacetimes to provide scales for the purpose of comparison, we did not need to make use of the particular structure of GR black hole spacetimes. As a result, our conclusions may be applied more generally to all gravitational theories that admit notions of energy conservation in the test-particle limit. Specifically, these include all geometric gravitational theories that admit stationary solutions, including all of the $f(R)$ theories and black hole alternatives that exist within the context of GR. As a consequence, we cannot yet say that $\mathrm{Sgr} \mathrm{A}^{*}$ is described by a GR black hole despite being able to conclude that a horizon must exist.

\section{APPENDIX A}

\section{GRAVITATIONAL BINDING ENERGY IN STATIONARY SPACETIMES}

As material falls into the gravitational potential well induced by Sgr A* it necessarily converts a portion of its gravitational binding energy into luminosity. In Section 3 we simply parameterized the amount of liberated energy in terms of some $\Delta \epsilon_{g}$, finding it unnecessary to specify this function further. In this appendix we derive a general expression for the magnitude of the binding energy available. We necessarily assume that gravity is described by a metric theory, and that this theory admits a stationary solution. For simplicity, we will not explicitly discuss nonspherical solutions, however this makes no formal difference. We take the metric signature to be -+++ and choose units such that $G=c=1$.

The state of an infalling particle, characterized by its 4-momentum, $p^{\mu}$, can be used to determine the energy available to radiation as measured at infinity. Given an initial momentum for an infalling particle, $p_{i}^{\mu}$, the momentum of the radiated photon, $p_{\gamma}^{\mu}$, and the final particle momentum, $p_{f}^{\mu}$, conservation of momentum gives

$$
p_{\gamma}^{\mu}=p_{i}^{\mu}-p_{f}^{\mu} .
$$

The steady state of the spacetime implies that along geodesics (e.g., the null geodesic followed by the photon, or the free-fall of the gas particle) $p_{t}$ is explicitly conserved, corresponding to a conserved energy. Thus, the photon's energy at infinity is simply $\Delta \epsilon_{g} \equiv-p_{\gamma_{t}}=p_{f_{t}}-p_{i_{t}}$. Since this quantity is observed at infinity, where the spacetime is flat, we are guaranteed that this quantity is not affected by the choice of coordinates deep in the gravitational well. In addition, this has the virtue of being precisely what is measured.

We will consider two scenarios, corresponding to different choices of $p_{f}^{\mu}$. The first of these is the most extreme: the particle was initially at rest at infinity $\left(p_{i_{t}}=-m\right)$, fell to some radius and came to rest, with ${ }^{8}$

$$
p_{\mu}^{\mathrm{rest}}=\left(\frac{m}{\sqrt{-g^{t t}}}, 0,0,0\right)
$$

\footnotetext{
8 This corresponds to the momentum of an observer that has freely fallen from infinity and decelerated only along the direction of motion. In the context of GR this choice of rest frame corresponds to the Zero Angular Momentum Observer for rotating black holes; however, this expression is completely general.
} 
at which point its accumulated binding energy was emitted. The available energy, per unit mass is then

$$
\Delta \epsilon_{g}=\frac{p_{t}-p_{t}^{\text {rest }}}{m}=\frac{\sqrt{-g^{t t}}-1}{\sqrt{-g^{t t}}}=\frac{z}{z+1},
$$

where the redshift is defined in the usual way: $z \equiv \sqrt{-g^{t t}}-1$.

Our second scenario involves the presence of an accretion disk. Previously, we assumed that the accreting material has no angular momentum, and therefore explicitly ignored orbital motion. Generally, the gas is expected to orbit the black hole, and the kinetic energy in the orbital motion will be supplied by the liberated gravitational binding energy, decreasing the reservoir of energy available to radiation. Hence we have placed a firm upper limit upon the luminosity of the accretion flow itself. However, we can compute the decrease explicitly for the simple case of stationary, spherically symmetric spacetimes. If

$$
d s^{2}=g_{t t} \mathrm{~d} t^{2}+g_{r r} \mathrm{~d} r^{2}+r^{2} \mathrm{~d} \Omega^{2},
$$

for orbits in the equatorial plane, $p_{t}^{\text {orbit }}$ and $p_{\phi}^{\text {orbit }}$ are conserved, $p_{\text {orbit }}^{\theta}$ vanishes due to symmetry and

$$
p_{\text {orbit }}^{r}=m \frac{\mathrm{d} r}{\mathrm{~d} \tau}=\sqrt{-\frac{1}{g_{r r}}\left(m^{2}+g^{t t} p_{t}^{\mathrm{orbit}^{2}}+\frac{p_{\phi}^{\mathrm{orbit}^{2}}}{r^{2}}\right)}=0 .
$$

If the orbit is to remain closed, we also require

$$
m \frac{\mathrm{d}^{2} r}{\mathrm{~d} \tau^{2}}=-\frac{1}{2} \frac{\partial}{\partial r} \frac{1}{g_{r r}}\left(m^{2}+g^{t t} p_{t}^{\text {orbit }}{ }^{2}+\frac{p_{\phi}^{\text {orbit }^{2}}}{r^{2}}\right)=0 .
$$

Solving these for $p_{t}^{\text {orbit }}$ and $p_{\phi}^{\text {orbit }}$ gives

$$
\begin{aligned}
p_{t}^{\text {orbit }} & =\frac{1}{\sqrt{-g^{t t}}} / \sqrt{1-\frac{1}{2} \frac{\partial \ln \left(-g^{t t}\right)}{\partial \ln r}} \\
& =\frac{1}{z+1} / \sqrt{1+\frac{\partial \ln (z+1)}{\partial \ln r}},
\end{aligned}
$$

which is generally larger than for the zero-angular momentum case (since $z$ generally decreases with radius). Correspondingly, $\Delta \epsilon_{g}$ in this case is generally lower.

While our discussion of the liberated gravitational binding energy is quite general, requiring only that gravity is described by a metric theory that admits a stationary solution, GR can provide some intuition regarding the magnitude of energy that can reasonably be released. Figure 2 shows the specific binding energy released by material as a function of radius. Typically, the ISCO is the final radius at which matter can efficiently radiate since inside this point material plunges into the black hole on a free fall timescale, ${ }^{9}$ rapid in comparison to the relevant radiative timescale for the rather pedestrian densities and magnetic fields

\footnotetext{
9 In the context of thin disks this corresponds to the zero-torque inner-boundary condition, which has been well tested by the fitting of X-ray spectra from X-ray binaries. In the context of thick disks, or quasi-spherical accretion flows, in principle magnetic fields can couple material inside of the ISCO to the flow outside, transferring energy in the process. However, thick disks are thick because $\eta_{r} \lesssim 0.1$, limiting the ability of the disk to cool efficiently. Thus, even in the presence of substantial magnetic coupling, thick disks are also bounded by this limit.
}

observed in Sgr A*. ${ }^{10}$ As a consequence, even if $100 \%$ of the gravitational binding energy released outside of the ISCO is radiated or goes into outflows, the maximum fraction of the rest mass that can be emitted is shown by the blue line in Figure 2. If the region where the accreted material finally comes to rest lies within the ISCO, additional gravitational binding energy will be released, and must subsequently be radiated in the stopping region. Thus, the radiative efficiency of the accretion flow, defined by

$$
\eta_{r}+\eta_{k} \equiv \frac{L_{\mathrm{acc}}}{\Delta \epsilon_{g}(R) \dot{M}}+\frac{L_{\mathrm{out}}}{\Delta \epsilon_{g}(R) \dot{M}},
$$

must satisfy

$$
\eta_{r}+\eta_{k} \leqslant \frac{\Delta \epsilon_{g}\left(r_{\mathrm{ISCO}}\right)}{\Delta \epsilon_{g}(R)}
$$

where $R$ is the radius of the stopping region. This limit shown as a function of $R$ in Figure 3 for zero-angular momentum accretion flows and accretion disks in Schwarzschild and Kerr spacetimes. Generally, once $R$ is constrained to lie within the photon orbit, the maximum $\eta_{r}+\eta_{k}$ possible is $91 \%$, corresponding to a zero-angular momentum accretion flow in a rapidly rotating $(a=0.998)$ Kerr spacetime. When an accretion disk is present this limit declines to $33 \%$. For the Schwarzschild spacetime, $\eta_{r}+\eta_{k}$ is bounded from above by $43 \%$ (zero-angular momentum accretion) and $14 \%$ (accretion disk). In all cases, these maximum values are substantially smaller than the limits set by recent submillimeter and infrared observations if Sgr $\mathrm{A}^{*}$ did not have a horizon.

\section{APPENDIX B}

\section{APPARENT SIZES OF COMPACT OBJECTS}

Observations of the size of a compact emitting region are necessarily impacted by strong gravitational lensing. In metric theories of gravity, objects associated with deep potential wells will appear larger to observers at infinity. The apparent size of the region is directly related to both the physical size and the redshift of the compact object. Thus, relating the actual size of a compact emitting region to the observed size requires some understanding of the spacetime structure around the object. This is, of course, one of the reasons we chose to cast the constraints upon the existence of a horizon, described in Section 3, in terms of $R_{a}$ and not a physical object size. Nevertheless, for completeness, we discuss the procedure here.

It is typically very difficult to compute the relationship between the physical and observed size and shape of a compact emitting object. However, in the special case of a spherically symmetric spacetime, this is generally tractable, independent of the particular form of the metric. We assume

$$
\mathrm{d} s^{2}=g_{t t} \mathrm{~d} t^{2}+g_{r r} \mathrm{~d} r^{2}+r^{2} \mathrm{~d} \Omega^{2},
$$

\footnotetext{
${ }^{10}$ In order to reach $\eta_{r}+\eta_{k} \simeq 1$ the energy of the accreting material must be extracted over the timescale comparable to the free-fall timescale at the point where $\eta_{r}+\eta_{k}$ of the binding energy has been released. That is, because the liberated binding energy rapidly increases as material falls inward, $\eta_{r}+\eta_{k}$ is determined by the last radius at which energy extraction can keep pace with the infall. Explicitly, for a Schwarzschild black hole this implies that the cooling timescale must be less than $\left(1-\eta_{r}-\eta_{k}\right) \Delta \epsilon_{g} /\left(\mathrm{d} \Delta \epsilon_{g} / \mathrm{d} \tau\right)=$ $2 R(R / 2 M)^{3 / 2} \sqrt{1-2 M / R}\left(1-\eta_{r}-\eta_{k}\right)$. For $\eta_{r}+\eta_{k}=99.6 \%$, this corresponds to cooling timescales of $2 \mathrm{~s}$ at $R=6 M$ and $0.3 \mathrm{~s}$ at $R=3 M$, both of which are only realized in practice in extraordinarily dense environments such as newly formed neutron stars.
} 
where $g_{t t}$ and $g_{r r}$ are functions of $r$ alone. Then, in the equatorial plane, the null geodesics are defined by

$$
\begin{aligned}
\frac{\mathrm{d} t}{\mathrm{~d} \lambda} & =g^{t t}, \\
\frac{\mathrm{d} r}{\mathrm{~d} \lambda} & =\sqrt{\frac{1}{g_{r r}}\left(-g^{t t}-\frac{b^{2}}{r^{2}}\right)} \\
& =\sqrt{\frac{-g^{t t}}{g_{r r}}\left[1-\frac{b^{2}}{(z+1)^{2} r^{2}}\right],} \\
\frac{\mathrm{d} \theta}{\mathrm{d} \lambda} & =0
\end{aligned}
$$

and

$$
\frac{\mathrm{d} \phi}{\mathrm{d} \lambda}=\frac{b}{r^{2}},
$$

where the equations for $\mathrm{d} t / \mathrm{d} \lambda$ and $\mathrm{d} \phi / \mathrm{d} \lambda$ are associated with the existence of a time-like and azimuthal Killing vectors, respectively, the equation for $\mathrm{d} \theta / \mathrm{d} \lambda$ is fixed by vertical symmetry and the equation for $\mathrm{d} r / \mathrm{d} \lambda$ arises from the null ray condition. In these $b$ is the impact parameter at infinity and $\lambda$ is an arbitrary affine parameter. The minimum radius reached by a given null geodesic occurs at its inner turning point, at which $b=r \sqrt{-g^{t t}}=r(z+1)$. Alternatively, this corresponds to the maximum $b$ that a null geodesic can have and still impact a surface of radius $r$. Thus, the apparent radius, $R_{a}$, of an object with physical radius $R$ is simply

$$
R_{a}=R(z+1) .
$$

Some care must be taken, however, when $R$ is smaller than the photon orbit. This is because rays which cross the photon orbit have no radial turning points, and therefore will in all cases be captured. This is clear from the definition of the photon orbit, $r_{\gamma}$ :

$$
\left.\frac{\mathrm{d}}{\mathrm{d} r} \frac{1}{(z+1)^{2} r^{2}}\right|_{r_{\gamma}}=0
$$

which corresponds to the position of the maximum of the "effective potential" in the radial equation. Thus, if

$$
b<r_{\gamma}\left[z\left(r_{\gamma}\right)+1\right] \Rightarrow \frac{b^{2}}{(z+1)^{2} r^{2}}<1 \text { for all } r .
$$

As a consequence, the apparent radius of objects for which $R<r_{\gamma}$ is the same as that for objects with $R=r_{\gamma}$. Thus, generally,

$$
R_{a}= \begin{cases}r_{\gamma}\left[z\left(r_{\gamma}\right)+1\right] & \text { if } \quad R \leqslant r_{\gamma} \\ R[z(R)+1] & \text { otherwise. }\end{cases}
$$

In the case of the Schwarzschild metric, this gives the well known result

$$
R_{a}= \begin{cases}3 \sqrt{3} M & \text { if } \quad R \leqslant 3 M \\ R \sqrt{\frac{R}{R-2 M}} & \text { otherwise. }\end{cases}
$$

For a rapidly rotating Kerr spacetime, the apparent radius in the equatorial plane may also be computed without undue difficulty (though in this case care must be taken into account for the nondiagonal components of the metric). Generally, this is given by

$$
R_{a}=\frac{1}{2}\left(b_{+}-b_{-}\right),
$$

$$
\begin{aligned}
b_{ \pm}= & \max \left(R \frac{R \sqrt{R^{2}-2 M R+a^{2}} \mp 2 a M}{R^{2}-2 M R},\right. \\
& \left.r_{ \pm \gamma} \frac{r_{ \pm \gamma} \sqrt{r_{ \pm \gamma}^{2}-2 M r_{ \pm \gamma}+a^{2}} \mp 2 a M}{r_{ \pm \gamma}^{2}-2 M r_{ \pm \gamma}}\right)
\end{aligned}
$$

in which $r_{ \pm \gamma}$ is the radius of the prograde/retrograde photon orbit. Since these differ for rotating black holes, we generally have three conditions. In the case of a maximally rotating black hole $(a=1)$, this expression is especially simple:

$$
R_{a}= \begin{cases}\frac{9}{2} M & \text { if } \quad R \leqslant r_{+\gamma} \\ \frac{R+8 M}{2} & \text { if } \quad r_{+\gamma}<R \leqslant 4 M \\ R \frac{R-1}{R-2} & \text { otherwise, }\end{cases}
$$

where $R$ is the object radius in Boyer-Lindquist coordinates. While $r_{+\gamma}=M$ in these coordinates for $a=1$, there remains a finite proper distance between the photon orbit and the horizon, the equality being an artifact of the coordinates themselves. For this reason, we distinguish between these formally, though it makes no difference (since $R_{a}=9 / 2$ for all $R$ between $r_{+\gamma}$ and the horizon), as it must not given that $R_{a}$ is a gauge invariant quantity as a consequence of its definition.

Most important for the present discussion is the fact that $R_{a}$ for the Schwarzschild and equatorial Kerr spacetimes differs by only about $15 \%$. Thus, despite the vastly different coordinate sizes, an object with $R=1 M$ embedded in a maximal Kerr spacetime has roughly the same apparent size as an object with $R=3 M$ embedded in a Schwarzschild spacetime. As a consequence, if the present limit upon the size of the submillimeter emitting region in $\mathrm{Sgr}^{*} *$ of $37 \mu$ as $\left(R_{a}=3.5 \mathrm{M}\right)$ is interpreted as a photosphere surrounding the stopping region, it constrains the size of a central emitting region to lie well within the photon orbit of both a Kerr and Schwarzschild black hole.

On the other hand, the anomalously small apparent radius might appear unphysical within the context of GR. However, this is easily rectified if the emission region is interpreted instead as the visible arc of an oncoming accretion disk (as a result of Doppler boosting and Doppler shifts, the receding side being considerably dimmer for the same reason; Broderick \& Loeb 2006a). While the equatorial extent of the arc can be significantly smaller than the minimum apparent radius in this situation, the vertical extent is still roughly $2 R_{a}$ (see, e.g., Broderick et al. 2009; Broderick \& Narayan 2006). Hence, unless the projected baseline was extraordinarily fortuitously aligned, again we would expect large measured sizes for central emission regions larger than the photon orbit radius. It is possible to coincidentally fit the existing spectral, polarization, and millimeter-VLBI observations using orbiting accretion flow models (Broderick et al. 2009). However, this is due at least in part to the fact that the existing millimeter-VLBI size constraint is essentially restricted to the east-west direction. Future millimeter-VLBI observations will be critical to unambiguously determining the morphology of the emitting region (Fish et al. 2009).

where 


\section{REFERENCES}

Agol, E. 2000, ApJ, 538, L121

Aitken, D. K., et al. 2000, ApJ, 534, L173

Blandford, R. D., \& Begelman, M. C. 1999, MNRAS, 303, L1

Bower, G. C., Wright, M. C. H., Falcke, H., \& Backer, D. C. 2003, ApJ, 588, 331

Bower, G. C., et al. 2004, Science, 304, 704

Bower, G. C., et al. 2006, ApJ, 648, L127

Broderick, A., \& Loeb, A. 2009, ApJ, 697, 1164

Broderick, A. E., Fish, V. L., Doeleman, S. S., \& Loeb, A. 2009, ApJ, 697, 45

Broderick, A. E., \& Loeb, A. 2005, MNRAS, 363, 353

Broderick, A. E., \& Loeb, A. 2006a, ApJ, 636, L109

Broderick, A. E., \& Loeb, A. 2006b, MNRAS, 367, 905

Broderick, A. E., \& Narayan, R. 2006, ApJ, 638, L21

Broderick, A. E., \& Narayan, R. 2007, Class. Quantum Grav., 24, 659

Doeleman, S. S., et al. 2008, Nature, 455, 78

Falcke, H., Melia, F., \& Agol, E. 2000, ApJ, 528, L13

Fish, V. L., Broderick, A. E., Doeleman, S. S., \& Loeb, A. 2009, ApJ, 692, L14

Ghez, A. M., et al. 2005, ApJ, 635, 1087

Ghez, A. M., et al. 2008, ApJ, 689, 1044

Gillessen, S., et al. 2009, ApJ, 692, 1075
Hawking, S. W. 2005, Phys. Rev. D, 72, 084013

Heger, A., et al. 2003, ApJ, 591, 288

Hornstein, S. D., et al. 2007, ApJ, 667, 900

Kato, S., Fukue, J., \& Mineshige, S. 2008, Black-Hole Accretion Disks (Kyoto: Kyoto University Press)

Krichbaum, T. P., et al. 2006, J. Phys. Conf. Ser., 54, 328

Macquart, J.-P., et al. 2006, ApJ, 646, L111

Marrone, D. P., Moran, J. M., Zhao, J.-H., \& Rao, R. 2006, ApJ, 640, 308

Marrone, D. P., Moran, J. M., Zhao, J.-H., \& Rao, R. 2007, ApJ, 654, L57

Mathur, S. D. 2009, in Physics of Black Holes: A Guided Tour, ed. E.

Papantonopoulos (Berlin: Springer-Verlag), 3

McClintock, J. E., Narayan, R., \& Rybicki, G. B. 2004, ApJ, 615, 402

Moneti, A., et al. 2001, A\&A, 366, 106

Narayan, R., Garcia, M. R., \& McClintock, J. E. 1997, ApJ, 478, L79

Narayan, R., \& Heyl, J. S. 2002, ApJ, 574, L139

Narayan, R., \& McClintock, J. E. 2008, New Astron. Rev., 51, 733

Narayan, R., Yi, I., \& Mahadevan, R. 1995, Nature, 374, 623

Oppenheimer, J. R., \& Snyder, H. 1939, Phys. Rev., 56, 455

Penrose, R. 1965, Phys. Rev. Lett., 14, 57

Quataert, E., \& Gruzinov, A. 2000, ApJ, 545, 842

Schödel, R., et al. 2007, A\&A, 462, L1

Shen, Z.-Q., et al. 2005, Nature, 438, 62

Wheeler, J. A. 1966, ARA\&A, 4, 393 